Gynäkologe 2015 • 48:276-277

DOI 10.1007/s00129-014-3363-7

Online publiziert: 25. Februar 2015

(c) Springer-Verlag Berlin Heidelberg 2015

G. Gille ${ }^{1}$ A. Schwenkhagen ${ }^{2}$

${ }^{1}$ Ärztliche Gesellschaft zur Gesundheitsförderung e.V., Lüneburg

${ }^{2}$ Hamburg

\title{
Junge Mädchen in der gynäkologischen Praxis
}

Mädchen im Sinne von „weiblichem Körperwissen“ und insbesondere von „fertility awareness" ein Recht hat.

Nur die Frauenärztin und der Frauenarzt wissen, wann Menstruationsbeschwerden das normale Maß überschreiten, weil nur sie Ursache und Wirkung in besonderer Weise überblicken können. Mit dem Beitrag von S. Anthuber und C. Deppe soll ein Bewusstsein für den achtsamen und kompetenten Umgang vor allem auch mit der Pathologie der Menstruation erzielt werden.

Ergänzend hierzu befasst sich $J$. Neulen in der Rubrik „Frauenheilkunde in der Praxis" mit der Pathophysiologie und dem therapeutischen Management der juvenilen Meno-/Metrorrhagie.

Eine brisante Mischung aus massenmedialer Stimulation, Halbwissen und Beziehungssehnsucht hat dazu geführt, dass auch sehr junge Mädchen sexuelle Kontakte aufnehmen - mit der potenziellen Gefahr gravierender gesundheitlicher Schäden und unter Umständen auch mit Konsequenzen für den gesamten Lebensentwurf. Die Vorgabe, dass eine Schwangerschaft unangebracht ist, ist unter Minderjährigen weitgehend unbestritten. Junge Mädchen wünschen sich deshalb zur Trennung von Sexualität und Fortpflanzung eine sichere, risikoarme und einfach zu handhabende Empfängnisverhütungsmethode - allerdings ohne Genaueres über ihre Wirkungsweise zu wissen und oft genug auch ohne ihr wirklich zu trauen. Mit Aufnahme des ersten Geschlechtsverkehrs wird also aus frauenärztlicher Sicht ein wichtiges Kapitel der Gesundheitsbiographie von Mädchen geschrieben. P. Oppelt bezieht zum Konsultationsanlass Kontrazeption aktuell Stellung.
Egal aus welchem Anlass ein junges Mädchen die gynäkologische Praxis aufsucht, immer bietet sich im Hinblick auf die Mädchengesundheit ein eindrucksvolles Angebot zur Prävention. Ergänzend zur Sexualerziehung in Schulen, bei der hauptsächlich biologisches Grundwissen vermittelt wird, ist es wichtig, Jugendliche in einem ganzheitlichen Verständnis von Sexualität kompetent und behutsam über die komplexen Veränderungen ihres Körpers aufzuklären. Frauenärzte können Mädchen aber auch beim Eintritt in die erwachsene Welt der Sexualität beratend zur Seite stehen und sie dabei unterstützen, Kompetenzen zu entwickeln, um diese Informationen zu nutzen. G. Gille zeigt auf, wie ärztliche Kompetenz, Glaubwürdigkeit und Verschwiegenheit die Frauenärztin und den Frauenarzt zum präferierten Vermittler präventiver Inhalte machen, damit jugendliche Sexualität nicht schon auf der Wissensebene scheitert.

\section{\) Frauenärzte können Mädchen bei der Entwicklung von Kompetenzen unterstützen}

Außerdem bietet die Frauenheilkunde Einflussmöglichkeiten, die Knochengesundheit junger Mädchen präventiv zu schützen, damit es im optimalen Zusammenspiel der Komponenten Genetik, Ernährung, Bewegung und endokriner Faktoren zur Ausbildung einer hohen Knochenmasse sowie der notwendigen Knochenarchitektur kommt, welche für den Rest der Lebens ausschlaggebend ist (I. Kyvernitakis und P. Hadji).

Für die Behandlung junger Mädchen in der frauenärztlichen Sprechstunde ist 
ein juristisches Grundwissen hilfreich und notwendig, um einerseits den Besonderheiten gerecht zu werden, die sich bei Patientinnen unter 18 Jahren ergeben, aber auch um sich selber in dieser Situation rechtlich korrekt abzusichern. Dafür vermittelt $C$. Halstrick profundes juristisches Basis-Know-how für die Mädchensprechstunde anhand von Fallbeispielen.

Wir sind davon überzeugt, dass die Frauenheilkunde das Potenzial hat, Mädchen entwicklungsbegleitend die Chance zu geben, gynäkologische Zusammenhänge positiv in ihre weibliche Identität integrieren und dadurch ihren Körper schätzen und schützen zu können. Aber junge Mädchen bedürfen auch bereits der konsequenten ärztlichen Diagnostik und Therapie bei unterschiedlichsten Beschwerden. Sich bewusst als Hausarzt der Mädchen und jungen Frauen im umfassenden Sinne zu positionieren, ist eine zutiefst frauenärztliche Aufgabe.

Dass dieses Themenheft das Seine da$\mathrm{zu}$ beitragen möge, wünschen sich und uns allen

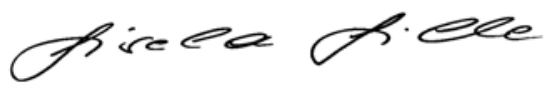

Dr. Gisela Gille
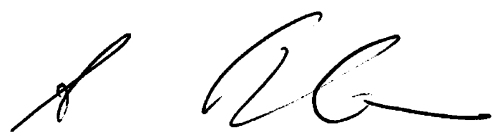

Dr. Anneliese Schwenkhagen

\section{Korrespondenzadresse}

\section{Dr. G. Gille}

Ärztliche Gesellschaft zur

Gesundheitsförderung e.V.

Drögenkamp 1, 21335 Lüneburg

gille@uni-lueneburg.de

Interessenkonflikt. G. Gille: Referentenhonorare von Gedeon Richter Pharma GmbH, Sanofi Pasteur MSD sowie Johnson \& Johnson GmbH Neuss. A. Schwenkhagen gibt an, dass kein Interessenkonflikt besteht.
F. Leidenberger, T. Strowitzki, 0. Ortmann (Hrsg.)

\section{Klinische Endokrinologie für Frauenärzte}

Springer-Verlag GmbH 2014, 5. Auflage, 691 S., (ISBN 978-3-642-38042-6), 199.00 EUR

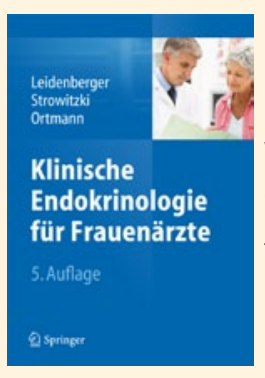

Das vorliegende Buch stellt die neu strukturierte und aktualisierte Version des seit der ersten Auflage im Jahre 1991 bewährten Klassikers der gynäkologischen Endokrinologie dar.

Gerade in einer immer stärker von Informationsflut und Schnelllebigkeit geprägten Zeit, hilft eine systematische und strukturierte Übersicht und Darstellung für das Verständnis des komplexen Systems der endokrinen Regelsysteme und ihrer assoziierten Störungen. Beginnend mit der Geschlechtsentwicklung über die reproduktive Lebensphase mit den durch Schwangerschaft und Stillzeit auftretenden Veränderungen sowie Übergang zum Senium werden die verschiedenen Lebensabschnitte durchschritten. Als Neuerung zu den vorausgegangenen Auflagen sind jeweils nach Darstellung der physiologischen Situation die klinisch relevanten pathophysiologischen Zustände in einem oder mehreren direkt anschließenden Kapiteln dargestellt. Interdisziplinäre Aspekte (z.B. Haut und Haare, Tumoren, Allgemeinerkrankungen, Umwelteinflüsse) werden durch zusätzlich eingefügte Kapitel aufgegriffen. Darüber hinaus finden sich im ersten Kapitel die Prinzipien der Hormonwirkung, sowie ein Glossar mit Erläuterung der wichtigsten Grundlagenbegriffe der Endokrinologie. In den Schlusskapiteln sind Grundlagen der Laboranalytik (inklusive Glossar) und die pharmakologischen Wirkprinzipien hormoneller Substanzgruppen dargestellt, sowie kommerziell erhältliche Hormonpräparate beziehungsweise Medikamente mit hormoneller Wirkung inklusive Dosierung tabellarisch aufgelistet - eine gute Option zum vergleichenden Nachschlagen trotz drohender zügiger Überholung durch neu auf den Markt drängende Generika-Präparate. Das Buch umfasst 21 Kapitel, welche jeweils mit einer Synopsis und Testfragen schließen. Der Textfluss wird dabei durch farbig hinterlegte Übersichten mit Zusammenfassung der vermittelten Fakten sowie durch Merksätze und zahlreiche Abbildungen und Tabellen aufgelockert. Zur Verschlankung wurden die Literaturangaben für alle Kapitel auf eine Online-Adresse („Springer-Link") ausgelagert, und sind dort durch Eingabe der ISBN problemlos abrufbar. In einem Service-Teil am Ende des Buches befinden sich die Lösungen der Multiple-Choice-Fragen sowie ein Stichwortverzeichnis mit Seitenangaben.

Den fachlich ausgewiesenen Autoren der einzelnen Kapitel kann attestiert werden, dass sie sowohl neue Erkenntnisse aus der Grundlagenforschung (z.B. Bedeutung des Kisspeptin-Systems für die GnRH-Sekretion), als auch eine praxis- und leitlinienorientierte therapeutische Umsetzung (z.B. Hormonelle Therapie) in den aktualisierten Inhalt haben einfließen lassen. Zum Erhalt der Übersichtlichkeit sind diese Einblicke verständlicherweise begrenzt, so dass der darüberhinausgehend interessierte Leser gegebenenfalls auf die Literaturzitate verwiesen werden muss. Über den Umfang der konservativen Kinderwunschtherapie bei Ovarfunktionsstörungen hinaus wurde auf die Darstellung der assistierten Reproduktionsverfahren und der modernen wohl bewusst verzichtet.

Zusammenfassend bietet das Werk für Berufsanfänger wie noch in der Ausbildung befindliche Ärzte eine exzellente Heranführung und zusammenfassende Darstellung aller relevanten Aspekte der gynäkologischen Endokrinologie. Aber auch für den bereits länger in der täglichen Praxis erfahrenen Kollegen empfiehlt sich das Buch zum erneuten Nachlesen pathophysiologischer Zusammenhänge und interdisziplinär überlappender Problemstellungen sowie von „state-of-the art"-Lösungshinweisen. Alles in allem eine wohltuende Lektüre für all jene, denen in der Zeit des allzeit verfügbaren „Stichwort-Wissens“ der Sinn nach einer zusammenhängenden, systematischen und umfassenden Wissensvermittlung mit echtem Erkenntnisgewinn steht!

\section{B. Sonntag (Hamburg)}

